\title{
Multi decision support model for Psychiatry Problem
}

\author{
A.Suhasini \\ Lecturer (SI.Gr.), Reader, \\ Professor \\ Department of Computer Science \\ and Engineering, \\ Annamalai University.
}

\author{
S.Palanivel \\ Lecturer (SI.Gr.), Reader, Professor \\ Department of Computer Science and \\ Engineering, \\ Annamalai University.
}

\author{
V.Ramalingam \\ Lecturer (SI.Gr.), Reader, Professor \\ Department of Computer Science and \\ Engineering, \\ Annamalai University
}

\begin{abstract}
Psychological distress and disabilities are increasingly identified among general population. Psychiatrist availability in rural areas is poor and often general practitioners have to identify and treat psychiatric problems like depression and anxiety. This work proposes a method to identify the psychiatric problems among patients using multi decision support system. Backpropagation (BP) and radial basis function (RBF) neural network models are used to design the decision support system. Forty four factors are considered for feature extraction. The features are collected from 400 patients and divided into four sets of equal size. Three sets of patient features are used to train the decision support system and one set of patient feature are used to evaluate performance of the system. Experimental results show that the proposed method achieves an accuracy of $98.75 \%$ for identifying the psychiatric problems.
\end{abstract}

Key Words: Multi decision support system, Backpropagation neural network, Radial basis function neural network, Psychiatry problem.

\subsection{Introduction}

On analyzing recent development, it becomes clear that the trend is to develop new method for decision making using computer in psychiatry and to evaluate these methods in practice. Medical expert system helps doctors to determine definitive diagnosis or a range of alternative diagnosis.

The conventional approach to build medical expert system requires the formulation of rules by which the input data can be analyzed. But the formulation of such rules is very difficult with large sets of input data. Realizing the difficulty, pattern classification technique such as artificial neural networks (ANN) has been applied as an alternative to conventional rule based expert system. ANN can be trained without encapsulating the knowledge derived from these rules. Hence ANN has been found to be more helpful than a traditional medical expert system in the diagnosis of diseases. For example, patients may not have similar signs and symptoms when disease is same. In addition, the disease of the patients cannot be classified into single class unless some more measurement and tests are made to solve ambiguity.

1.1 Overview of Psychiatry Problem

Computers could have a role in supplementing patient assessments, diagnosis and treatment in a variety of clinical settings [1]. A number of software tools are being designed and are already in use [16]. These tools are found useful in administrative, data management and in clinical experiments. These tools reduce time and minimize errors in medical practice. The clinical decision support system synthesize and integrate patient specific information, perform complex evaluations and present the results to clinicians in a timely fashion[3]. In India most of the health care burden is at the primary care level. The primary care physicians often have to manage variety of clinical decisions themselves [5]. Computers may be of immense benefit to them. Decision support systems may enable the doctor to assess his performance and improve his diagnostic accuracy. Psychiatric problems are very common and cause significant delay in recovery and often require costly investigations before coming to its diagnosis [12].

\subsection{Problems Faced in Clinical Decision Support Systems}

There are certain general problems in preparing clinical decision support systems (CDSS). It is not possible to create CDSS because there is no electronic medical records (EMR) at primary care level. Technologies are not yet widely available to most hospitals and many electronic data information standards not yet been adopted industrywide. One of the most difficult barriers is the development of a standardized EMR. There are no defined set of recommendations for an EMR and no industry-wide standard for EMR [9]. Due to the limited implementation of computer-assisted diagnostic and management tools only a few evaluations have been conducted and no definitive conclusions have been reached from the CDSS.

\subsection{Literature Review on Decision Support System}

Decision support system play a major role in health-care. There are only a few computerized clinical decision support systems for psychiatry problems. In [14], the authors have designed and developed a computerised decision support system for the treatment of major depressive disorder using evidence based guidelines. They have transferred the knowledge gained from the Texas medication algorithm project (TMAP). This computerised decision support system (compTMAP) provides support in diagnosis treatment follow up and preventive care. This system can be incorporated into the clinical setting.

Harmonex group of medical experts and software technologists have developed a system for the use of mental health settings. By using electronic medical records they have deviced decision supporting systems. The 
harmonex neuroscience research division (NSR) deals with schizophrenia, bipolar disorders, depression, anxiety, ADHD and other psychiatric syndromes.

A brazilian university group of psychiatrists have devised a system for diagnosing schizophrenia [11]. The system is called SADDESQ is modelled for the use of students in psychiatry to diagnose psychotic disorders. The knowledge is initially extracted from clinical interviews. These interviews are aimed to explore the experts decision making process. A graph methodology was employed to identify the elements involved in the reasoning process. This knowledge is organised and modeled by means of algorithms and transferred to computational model created by the covering approach.

\subsection{Decision Support System for Identifying the Psychiatry Problems}

The psychiatry problems among patients can be identified using multi decision support system. In this work, backpropagation and radial basis function neural networks are used to construct the system. Artificial neural networks (ANNs) are intelligent systems that are related to a simplified biological model of the human brain. They are composed of many simple elements, called neurons, operating in parallel and connected to each other in the forward path by some multipliers called the connection weights. Neural networks are trained by adjusting values of these connection weights between the network elements. Neural networks have self learning capability, are fault tolerant and noise immune, and have applications in system identification, pattern recognition, classification, speech recognition and image processing, etc. [15]. ANNs are potentially useful for studying the complex relationships between inputs and outputs of a system. Backpropagation and radial basis function neural networks are deserted in the following subsections.

\subsection{Backpropagation Neural Network (BPNN)}

Backpropagation neural network is a feed forward neural network used to capture the relationship between the inputs and outputs [10]. In this work, a three layer feedforward neural network as shown in Fig 1. is used with hyperbolic tangent $(\tanh ())$ as activation function. The neural network is trained using backpropagation algorithm [4].

In the backpropagation training algorithm the error in the output neuron $\mathrm{q}$ is given by

$$
\delta_{\mathrm{q}}=\mathrm{o}_{\mathrm{q}}\left(1-\mathrm{o}_{\mathrm{q}}\right)\left(\mathrm{t}_{\mathrm{q}}-\mathrm{o}_{\mathrm{q}}\right)
$$

where $\mathrm{o}_{\mathrm{q}}$ and $\mathrm{t}_{\mathrm{q}}$ are the actual and desired outputs of neuron $\mathrm{q}$ in the output layer, respectively. The weight from neuron $\mathrm{p}$ in the hidden layer to neuron $\mathrm{q}$ in the output layer is adjusted using

$$
\mathrm{w}_{\mathrm{pq}}(\mathrm{n}+1)=\mathrm{w}_{\mathrm{pq}}(\mathrm{n})+\eta \delta_{\mathrm{q}} \mathrm{o}_{\mathrm{p}}
$$

where $\eta$ is the learning rate coefficeint, $0<\eta<1$, and $\mathrm{w}_{\mathrm{pq}}(\mathrm{n})$ and $\mathrm{w}_{\mathrm{pq}}(\mathrm{n}+1)$ are the weights before and after adjustment, respectively. The error in the output layer is propagated backwards to adjust the weights in the hidden layers. The error in neuron $\$ \mathrm{p} \$$ in the hidden layer is obtained using

$$
\delta_{\mathrm{q}}=\mathrm{o}_{\mathrm{p}}\left(1-\mathrm{o}_{\mathrm{p}}\right) \Sigma_{\mathrm{q}} \delta_{\mathrm{q}} \mathrm{w}_{\mathrm{pq}}(\mathrm{n}+1)
$$

The error $\delta_{\mathrm{q}}$ is used to adjust the weights connecting to neuron $\mathrm{p}$ in the hidden layer. This process is repeated for all the hidden layers. Application of all inputs once to the network and adjusting the weights is called an epoch. In the backpropagation training algorithm the network weights are adjusted for certain number of epochs to map the relationship between inputs and outputs.

\subsection{Radial Basis Function Neural Network (RBFNN)}

The RBFNN [8] has a feedforward architecture with an input layer, a hidden layer, and an output layer as shown in Fig. 2. Radial basis functions are embedded into a twolayer feed forward neural network. Such a network is characterized by a set of inputs and a set of outputs. In between the inputs and outputs there is a layer of processing units called hidden units. Each of them implements a radial basis function. The input layer of this network has $n_{i}$ units for a $n_{i}$ dimensional input vector. The input units are fully connected to the $n_{h}$ hidden layer units, which are in turn fully connected to the $n_{c}$ output layer units, where $n_{c}$ is the number of output classes. The activation functions of the hidden layer are Gaussians, and are characterized by their mean vectors (centers) $\mu_{\mathrm{i}}$, and covariance matrices $C_{i}, i=1,2, \ldots \ldots, n_{h}$. For simplicity, it is assumed that the covariance matrices are of the form $\mathrm{C}_{\mathrm{i}}=$ $\sigma^{2} \mathrm{I}, \mathrm{i}=1,2, \ldots, \mathrm{n}_{\mathrm{h}}$.

Then the activation function of the $i^{\text {th }}$ hidden unit for an input vector $\mathrm{x}_{\mathrm{j}}$ is given by

$$
\left(-\left\|x_{j}-\mu_{i}\right\|^{2}\right) g_{i}\left(x_{j}\right)=\exp
$$

$2 \sigma^{2}{ }_{i}$

The $\mu_{\mathrm{i}}$ and $\sigma^{2}{ }_{\mathrm{i}}$ are calculated using k-means clustering algorithm. The algorithm is composed of the following steps:

1. Randomly initialize the input vectors to k-means (clusters) $\mu_{1}, \mu_{2}, \ldots, \mu_{\mathrm{k}}$

2. Classify $n_{k}$ input vectors according to nearest $\mu_{\mathrm{i}}$. 3. Recompute $\mu_{\mathrm{i}}$.

4. Repeat the steps 2 and 3 until no change in $\mu_{\mathrm{i}}$ The number of hidden units in the network and their spread influence the smoothness of the mapping. The assumption $\sigma_{i}^{2}=\sigma^{2}$ is made and is given in (5) to ensure that the activation functions are not too peaked or too flat.

$\sigma^{2}=\eta d^{2}---$

In (5) $\mathrm{d}$ is the maximum distance between the chosen centers, and $\eta$ is an empirical scale factor which serves to control the smoothness of the mapping function. Therefore, (4) can be written as

$$
\left(-\left\|x_{j}-\mu_{i}\right\|^{2}\right) g_{i}\left(x_{j}\right)=\exp
$$
$\eta d^{2}$

The hidden layer units are fully connected to the $n_{c}$ output layer units through weights $\mathrm{w}_{\mathrm{ik}}$. The output units are linear, and the response of the $\mathrm{k}^{\text {th }}$ output unit for an input $\mathrm{x}_{\mathrm{j}}$ is given by

$\mathrm{y}_{\mathrm{k}}\left(\mathrm{x}_{\mathrm{j}}\right)=\Sigma \mathrm{w}_{\mathrm{ik}} \mathrm{g}_{\mathrm{i}}\left(\mathrm{x}_{\mathrm{j}}\right), \mathrm{k}=1,2, \ldots, \mathrm{n}_{\mathrm{c}}$

where $g_{0}\left(x_{j}\right)=1$. Given $n_{t}$ feature vectors from $n_{c}$ classes, training the network involves computing $\mu_{\mathrm{i}}, \mathrm{i}=1,2, \ldots, \mathrm{n}_{\mathrm{h}}, \eta$, $\mathrm{d}$, and $\mathrm{w}_{\mathrm{ik}}, \mathrm{i}=0,1,2, \ldots, \mathrm{n}_{\mathrm{h}}, \mathrm{k}=1,2, \ldots, \mathrm{n}_{\mathrm{c}}$. The training procedure is given below: 
Determination of $\mu_{i}$ and $\mathbf{d}$ : Conventionally, the unsupervised k-means clustering algorithm [2], can be applied to find $n_{h}$ clusters from $n_{t}$ training vectors. In order to obtain clusters according to psychiatry problem (presence/absence) and (depression/anxiety/absence), the kmeans clustering is used in a supervised manner. Training features vectors belonging to the same psychiatry problem are clustered to $\mathrm{n}_{\mathrm{h}} / \mathrm{n}_{\mathrm{c}}$ clusters using k-means clustering algorithm. This is repeated for each psychiatry problem yielding $n_{h}$ clusters for $n_{c}$ psychiatry problems. These cluster means are used as the centers $\mu_{\mathrm{i}}$ of the Gaussian activation functions in the hidden layer. The parameter $d$ is then computed by finding the maximum distance between $\mathrm{n}_{\mathrm{h}}$ cluster means.

Determining the weights $w_{i k}$ between the hidden and output layer: Given that the Gaussian function centers and widths are computed from $n_{t}$ training vectors, (7) may be written in matrix form as

$\mathrm{Y}=\mathrm{GW}$

where $Y$ is a $n_{t} \times n_{c}$ matrix with elements $Y_{i j}=y_{j}\left(x_{i}\right), G$ is a $\mathrm{n}_{\mathrm{t}} \mathrm{x}\left(\mathrm{n}_{\mathrm{h}}+1\right)$ matrix with elements $\mathrm{G}_{\mathrm{ij}}=\mathrm{g}_{\mathrm{j}}\left(\mathrm{x}_{\mathrm{i}}\right)$, and $\mathrm{W}$ is a $\left(n_{h}+1\right) \times n_{c}$ matrix of unknown weights. $W$ is obtained from the standard least squares solution as given by

$W=\left(G^{\mathrm{T}} G\right)^{-1} G^{\mathrm{T}} Y$

To solve W from (9), G is completely specified by the clustering results, and the elements of $\mathrm{Y}$ are specified as

$$
Y_{i j}=\left\{1 \quad \text { if } x_{i} \in \text { class } j\right.
$$

$\{0$ otherwise

\subsection{Experimental Results}

In this work we have used a psychiatry database for analyzing the performance of the decision support system for identifying the psychiatry problems. The patients demographic data, social information's, past history of psychiatric morbidity, family history of psychiatric morbidity, nature and duration of unexplained medical symptoms, head and neck, chest, abdomen, genitor-urinary, central nerves system, general, care utilization, previous doctor consultations used as features. The complete list of 44 factors used for identifying the psychiatry problems is given in Appendix I. The database is created from 400 patients treated in Rajah Muthiah Medical College Hospital, Annamalai University, Tamilnadu, India. Database consists of patients who are referred to the psychiatric department by the doctors for assessment of psychiatric morbidity among patients with unexplained medical symptoms (UMS). The database is divided into four sets of equal size.

For each patient a value in the range (0-9) is obtained for each of the 44 factors. The psychiatrist administers hamilton score of anxiety and depression. He then interviews the patient and comes to a depression or anxiety diagnosis if there are any on each case.
The 44 features $\left(\mathrm{x}_{1}, \mathrm{x}_{2}, \ldots \ldots . \mathrm{x}_{44}\right)$ collected from the patient are normalized to $[-1,1]$ using

$$
y_{i}=\frac{2.0\left(x_{i}-x_{\min }\right)}{\left(x_{\max }-x_{\min }\right)}-1
$$

where $\mathrm{x}_{\max }$ and $\mathrm{x}_{\min }$ are the maximum and minimum values of the unnormalized features.The normalized features $\left(\mathrm{y}_{1}, \mathrm{y}_{2}, \ldots . . \mathrm{y}_{44}\right)$ are given as input to the neural networks. The presence $\left(\begin{array}{ll}1 & -1\end{array}\right)$ or absence $\left(\begin{array}{ll}-1 & 1\end{array}\right)$ of psychiatric problem is used as output. In an another experiment forty six features are used as inputs $\left.\mathrm{y}_{1}, \mathrm{y}_{2}, \ldots . . \mathrm{y}_{46}\right)$ and none $(1$ $1-1)$, depression (-1 $1-1)$ and anxiety $\left(\begin{array}{lll}-1 & -1 & 1\end{array}\right)$ are used as outputs, where $\mathrm{y}_{45}$ and $\mathrm{y}_{46}$ are the hamilton score of anxiety and depression, respectively.

In this work, initially the backpropagation and radial basis function neural networks are trained to produce the required output for the given input features. Three sets of data are used for training and one set is used for testing the neural networks. The performance indicators are accuracy (percentage of correct diagnoses), sensitivity and specificity. The sensitivity and specificity are given by

true

positive
sensitivity $=\quad$ - - - 100

false negative)

(true positive +

$$
\text { * } \begin{gathered}
\text { negative } \\
\text { specificity }=\quad \text { - } \\
\text { (true negative }+
\end{gathered}
$$

false positive)

\subsection{Identifying Psychiatry Problems using Backpropagation Neural Networks}

The three layer feedforward backpropagation neural network model used for identifying the psychiatry problem is shown in Fig. $\backslash \operatorname{ref}\{b p\}$. The following five backpropagation neural network structures are analyzed for detecting the presence or absence of psychiatry problems: S1 (44L-20N-10N-2L), S2 (44L-25N-15N-2L), S3 (44L$30 \mathrm{~N}-20 \mathrm{~N}-2 \mathrm{~L})$, S4 (44L-35N-25N-2L) and S5 (44L-40N$30 \mathrm{~N}-2 \mathrm{~L})$. The integer number in $44 \mathrm{~L}-20 \mathrm{~N}-10 \mathrm{~N}-2 \mathrm{~L}$ indicates the number of units/neurons used in each layer (input layer, hidden layer1, hidden layer2, and output layer, respectively) and $\mathrm{L}, \mathrm{N}$ denotes number of linear and nonlinear units, respectively. The nonlinear units use $\tanh (s)$ as the activation function, where $s$ is the activation value of the unit. The networks are trained for 2000 epochs. For training, three sets of features vectors are used. One set of feature vector is used for testing the network in rotation basis. The result are given in Fig 3. The network structure $44 \mathrm{~L} 20 \mathrm{~N} 10 \mathrm{~N} 2 \mathrm{~L}$ gives an accuracy of $98.93 \backslash \%$.

Similarly the following five structures are used for identifying the psychiatry problems such as depression, anxiety and absence of psychiatry problems: 
S1 (46L-20N-10N-3L), S2 (46L-25N-15N-3L), S3 (46L$30 \mathrm{~N}-20 \mathrm{~N}-3 \mathrm{~L})$,

S4 (46L-35N-25N-3L) and S5 (46L-40N-30N-3L). The result are shown in Fig. 4. The structure 46L 40N 30N 3L gives a performance of $98.70 \backslash \%$.

4.2 Identifying Psychiatry Problems using Radial Basis Function Neural Networks

The radial basis function neural network shown in Fig. 2 is used for identification. For training, the RBF centers are located using k-means algorithm for three sets of features vectors. The value $\mathrm{k}=1,2,3,4$ and 5 has been used in our studies. The number of neurons in the output layer is one for experiment 1 (presence/absence) and three for experiment 2 (depression/anxiety/absence). The weights in the RBFNN are determined using the least squares algorithm..

For testing, feature vector is given as input to the RBFNN model. For experiment 1 , if the output of the network is greater than a threshold then it represent the presence of psychiatry problems. For experiment 2, highest output is used to identify the type of psychiatry problems. The performance of RBFNN for different means are shown in Fig.5 and Fig. 6 for experiment 1 and experiment 2, respectively.

\section{0 Conclusion}

This work proposed a decision support system for identifying the psychiatric problem. The decision support system was designed using backpropagation and radial basis function neural networks. The decision support system combines the output of backpropagation and radial basis function neural networks using weighted sum rule. Experiment results show in Fig 7 and fig 8 show that the decision support system gives an accuracy of $98.75 \%$ for identifying the psychiatry problems.

\section{REFERENCES}

1. J.Bremer, E.V.Beresin, "Computer in Psychiatry today “ , Academic Psychiatry, 24(3) (2000), 168-172.

2. R.O.Duda, classification", P.E.Hart,D.G.Stork,"Pattern Newyork,2001.

3. D.L Hunt, R.B Haynes, "Effect of computer based clinical decision systems on physician performance and patient outcomes" Journal of American medical association, 280,(1998). 13391345.
4. S.Haykin,'Neural networks a comprehensive foundation", Pearson education, Asia,2003.

5. K.S.Jacob, "The diagnosis and management of depression and anxiety in primary care the need for a different framework " Postgraduate medical journal , 82,(2006),836-839.

6. K.S.Jacob, "A simple protocol to manage unexplained medical symptoms in medical practice " National mediacl journal of india, 17,(2004),326-328.

7. G.H.Lewis, A.Pelosi, R.Arya,"Measuring psychiatric disorder in the community: a standardized assesetmentfor lay interviewers", Psychological medicine,22,(1992),465-486.

8. S.R.Lian hock koha, Y.V Venkatesh, "An integrated automatic face detection and regnition system", Pattern Recognition, 35(6),(2002),12591273.

9. J.Morrisey,"Clinical- care it still the final frontier", postgraduate medical journal.

10. H.Poh, "A neural network approach for marketing strategies research and decision support", Ph.D. thesis, Stanford University,1991.

11. D.Razzouk, J.Mari, I.Shirakawa , J.Wainer, D.Sigulem, "Decision support system for the diagnosis of schizophrenia disorders", Brazilian journal of medical and biological research, 39(1),(2006),119-128.

12. P.Salmon, C.Dowrick, A.Ring, "Voice but unheared agendas:qualitative analysis of the psychosocial cues that patients with unexplained medical sysmtoms present to general practitioners", British journal of general practice, 164,(2004),171-176.

13. P.Salmon, C.Dowrick, C.Ring, "What do general practice want when they present with unexplained medical symptoms", Journal of psychosomatic research, 59,(2005),225-260.

14. M.H.Trivedi, J.K.Kern,B.D.Grannemann, K.Z Altshuler, P.Sunderajan,"A computerized clinical decision support system as a mean of implementing depression guide lines", Psychaitric services,55(8).(2004),879-885.

15. G.K.Venyagamoorthy, V.Moonasar, K.Sandrasegaran,"Voice Regnition using networks", in:proc. IEEE,(1998),29-32.

16. M.G.Weiner, E.Pifer, "Computerized Decision support and the Quality of care", Managed care magazine, 9(5),(2000), 302-208. 


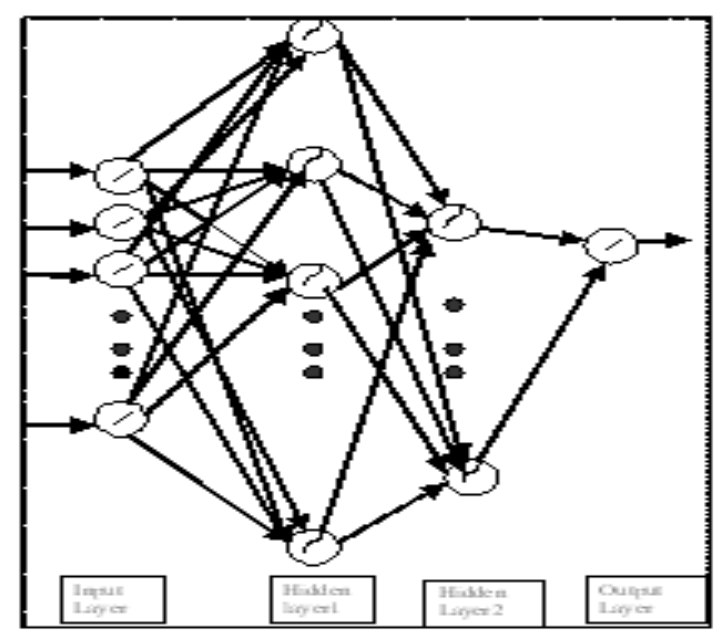

Fig 1 Backpropagation neural network

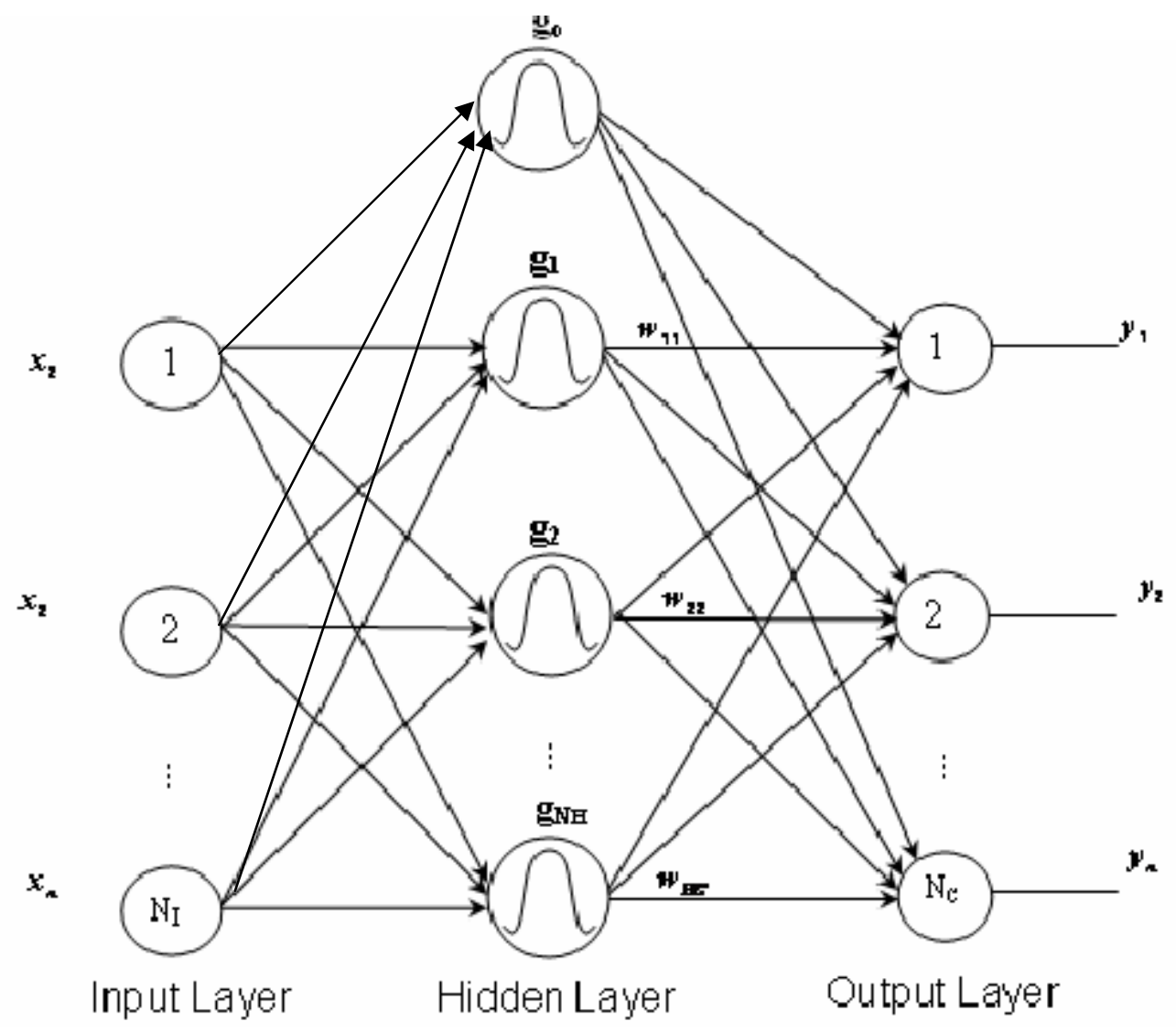

Fig 2 Radial basis function neural network 


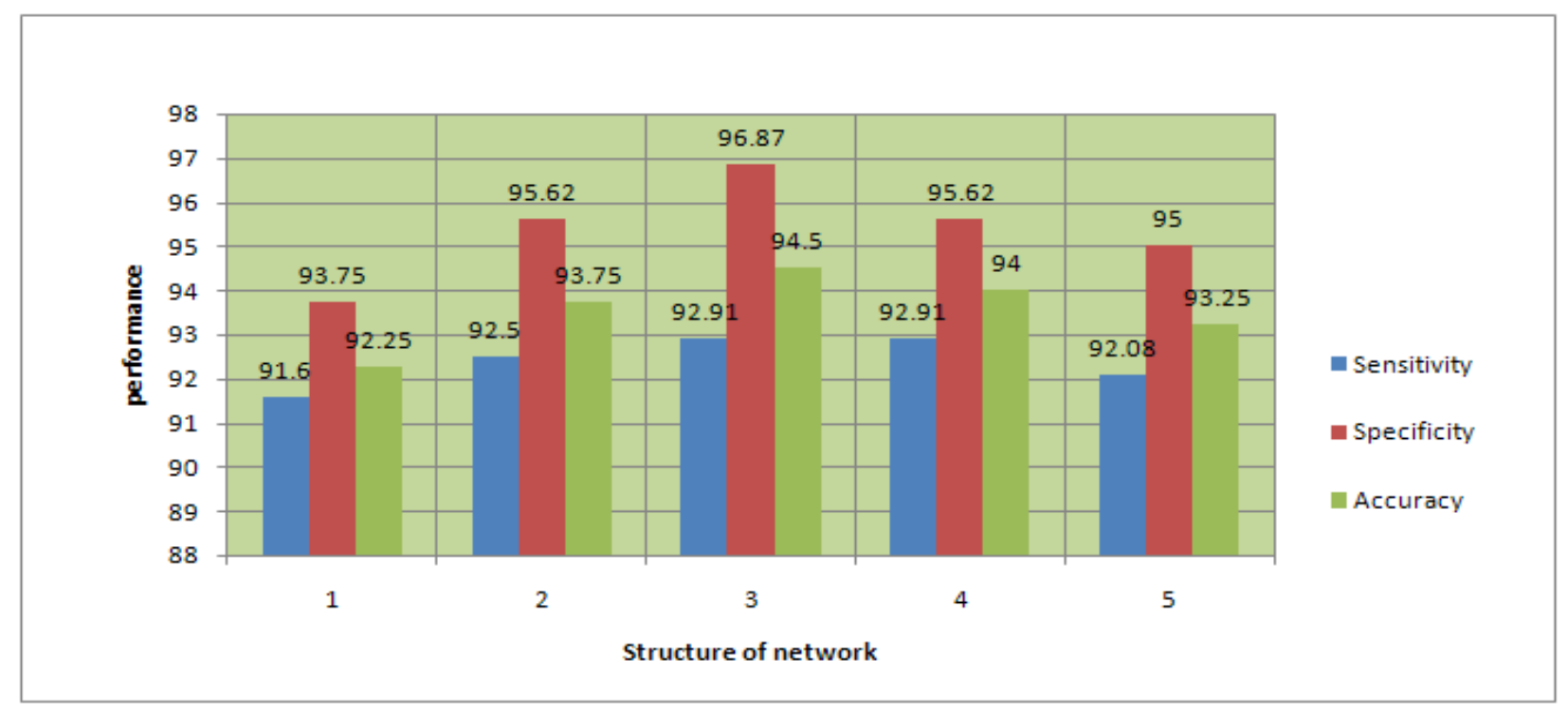

Fig 3 : Performance of BPNN for detecting the presence/absence of psychiatry problems

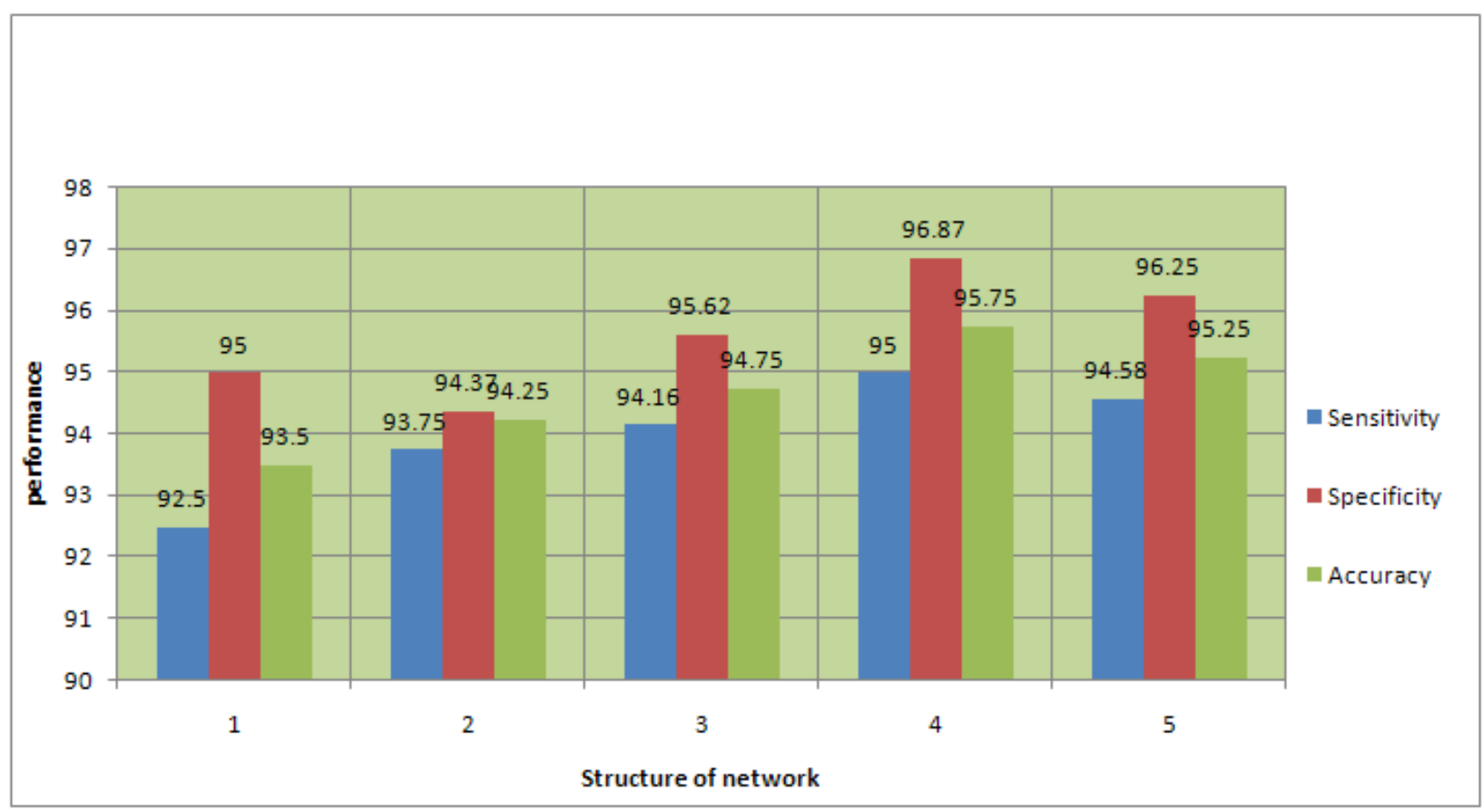

Fig 4. Performance of BPNN for identifying the psychiatry problems 


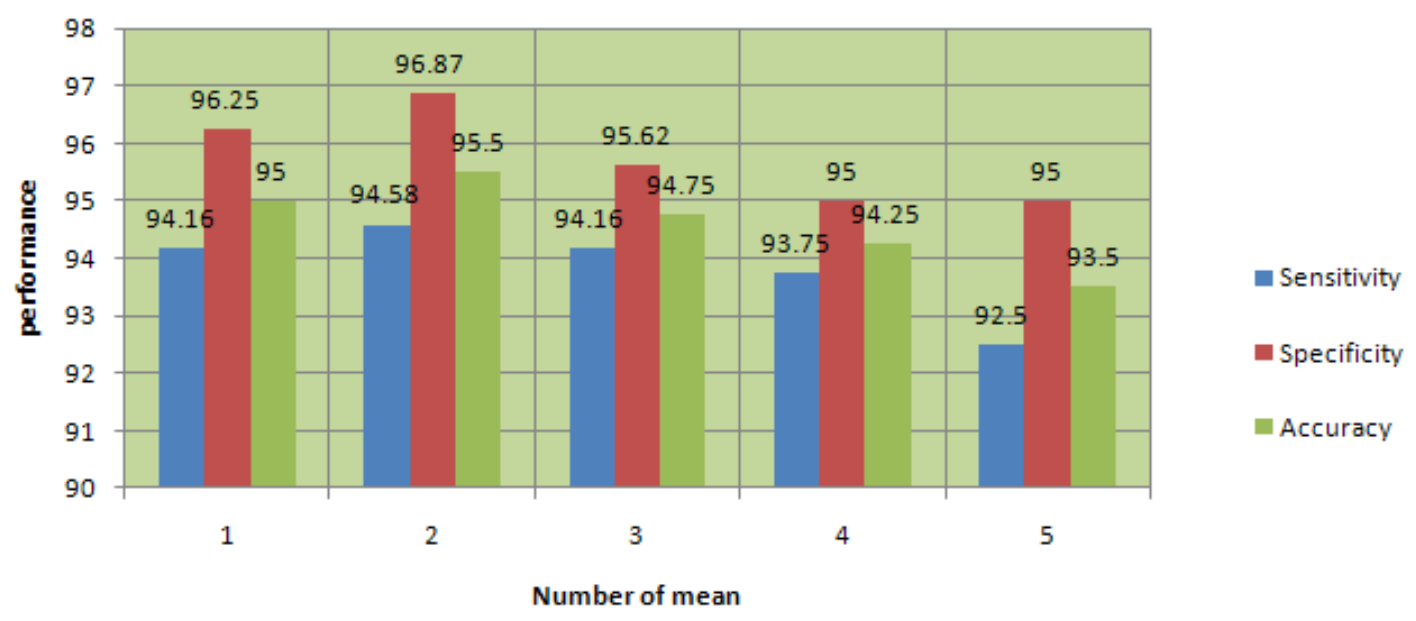

Fig 5 Performance of RBF for detecting the presence /absence of psychiatry problems

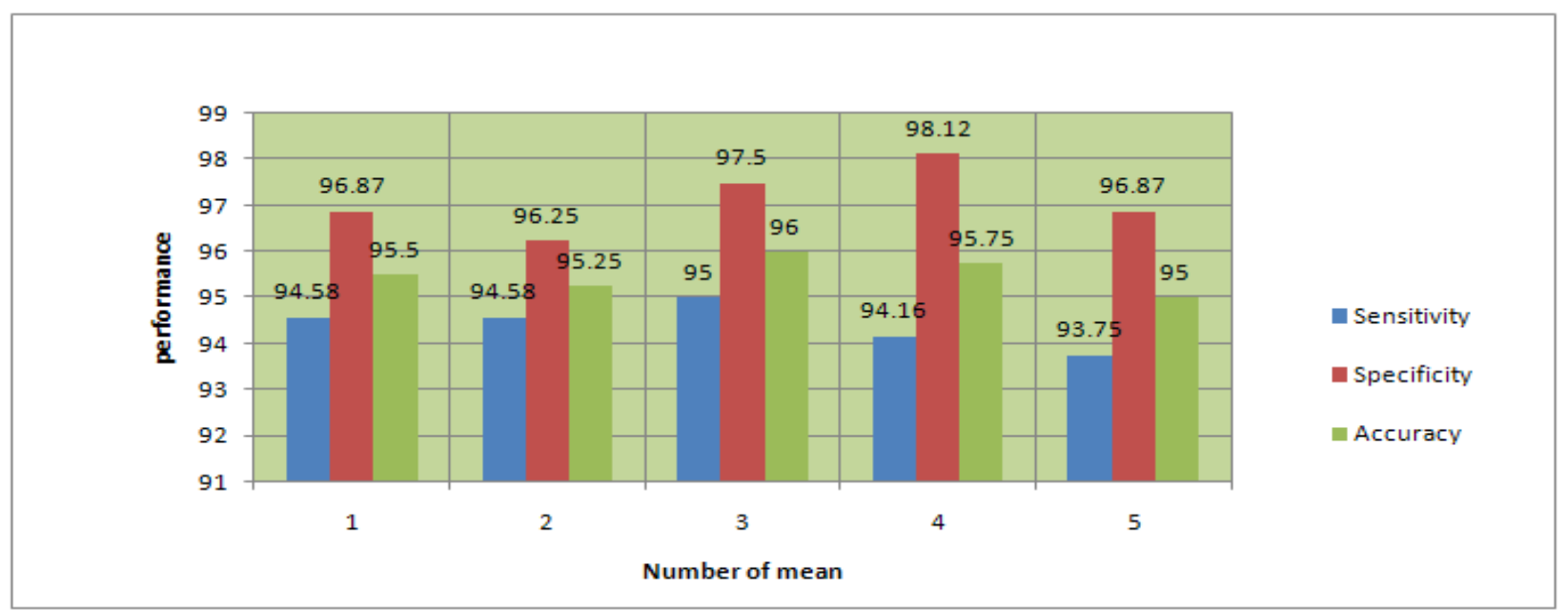

Fig 6 Performance of RBF of identifying the psychiatry problems. 


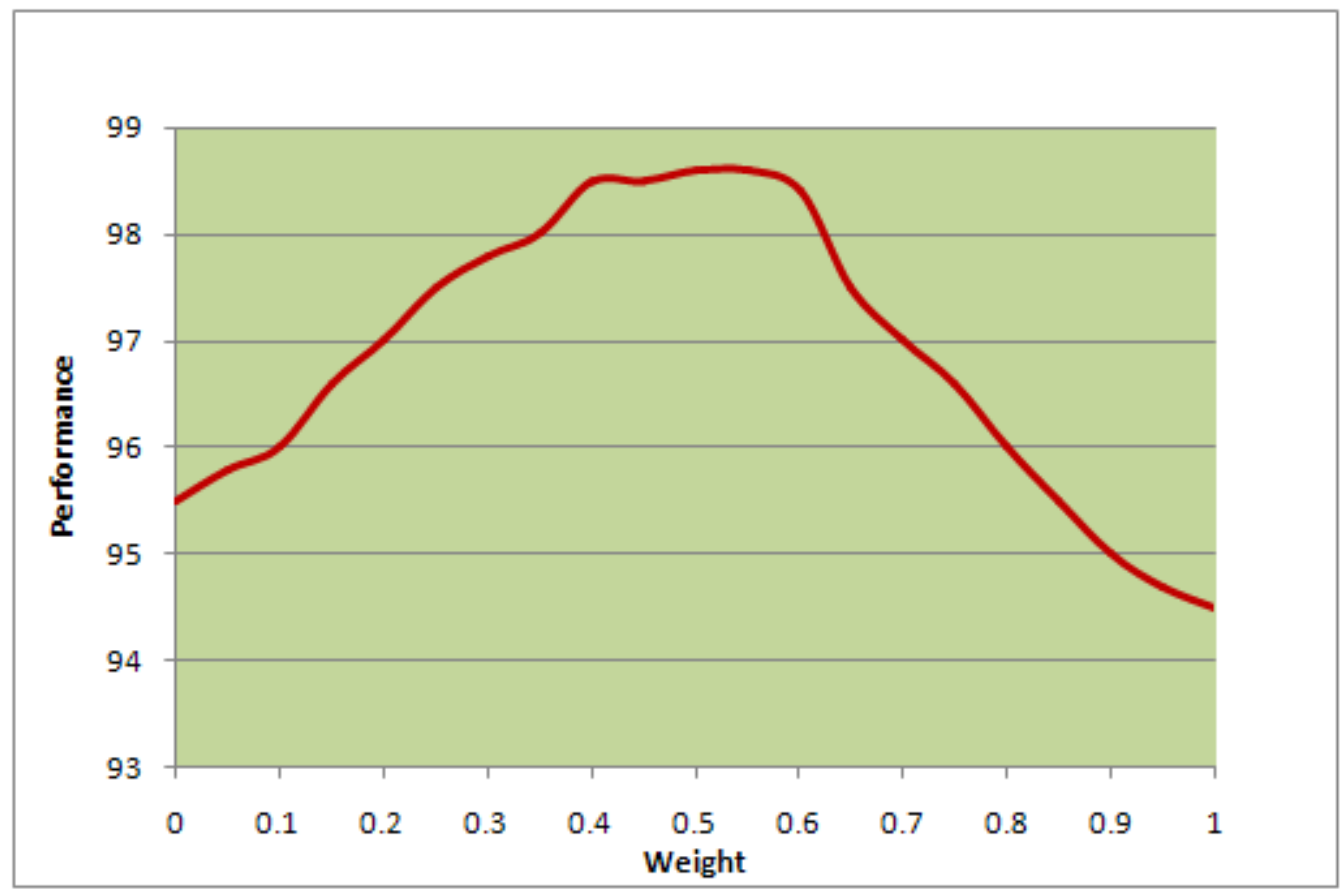

Fig 7 Performance of DSS for detecting the presence /absence of psychiatry problems 


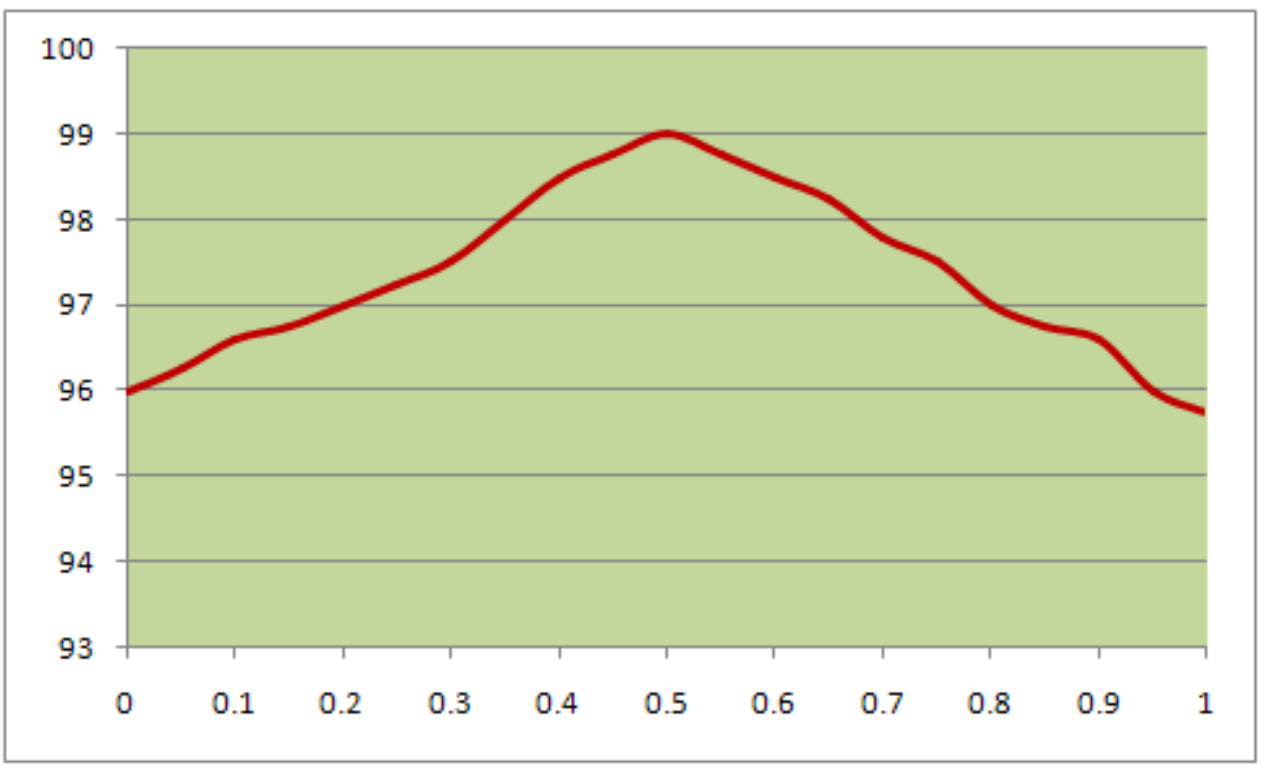

Fig 8 Performance of DSS for identifying of psychiatry problems

\section{Appendix I}

Fourty four factors used for identifying the psychiatry problems

1. Demographic and history: Age, Gender, Family history of Psychiatric problems, Past history of Psychiatric problems, Social support, Psychological symptoms, UMS.(7)

2. Head and neck : Crawling sensation in the scap, Tightness around head, Blurring of vision, Lack of smell, Lack of taste, Burning sensation in the eyes, Ball rolling in the throat,Dryness of throat and mouth.(8)

3. Chest : Choking feeling in breathing, Tightness around chest, Palpitations, Burning sensations in the chest, Difficulty in breathing.(5)

4. Abdomen : Vomiting, Hiccough, Churning sensation in abdomen, Indigestion, Constipation, Frequent belching, Poor apetite.(7)
5. Genitor-urinary: Frequent micturition, Loss of libido, Menstrual irregularity, Menstrual pain.(4)

6. Central nerves system : Dizziness, Nervous instability, Numbness over the body, Crawling sensation all over the body, Slowness of activity, Forgetfulness, Poor concentration, Tiredness-fatigue.(8)

7. General : Body pains, Back pain, Neck pain.(3)

8. Care utilization : Multiple symptoms, Frequent consultations.(2) 